\title{
Some Integrals Involving Associated Legendre Functions
}

\author{
By S. N. Samaddar
}

\begin{abstract}
Calculations of some uncommon integrals involving Legendre functions and their derivatives, which may not be readily evaluated using known results, are presented. Some results show a special type of orthogonality relation in a certain sense. A few of these integrals find their applications in diffraction or scattering problems.
\end{abstract}

1. Introduction. In the analysis [1] of the scattering of a cylindrical electromagnetic wave by a spherical object, one encounters some special type of integrals involving associated Legendre functions. In the process of computing these integrals, one finds a number of related integrals as by-products, which may not be readily evaluated using the known results. Once a few of these integrals are calculated, the rest may be found by using the recurrence relations or other known results. For the sake of briefness, the proofs of only the primary results will be sketched and the rest will simply be presented.

2. Some Preliminary Results. Let us consider the following recurrence relations [2]:

$$
\begin{aligned}
\left(1-x^{2}\right)^{1 / 2} \cdot P_{n}^{m-1}(x) & =\left[P_{n+1}^{m}(x)-P_{n-1}^{m}(x)\right] /(2 n+1) \\
& =\left[x P_{n}^{m}(x)-P_{n-1}^{m}(x)\right] /(n-m+1),
\end{aligned}
$$

where $m, n=0,1,2, \cdots$.

Then the following relation can easily be established:

$$
\begin{aligned}
& \int_{-1}^{1} \frac{x P_{n}^{m}(x) P_{n+2 l+1}^{m}(x) d x}{1-x^{2}}-\int_{-1}^{1} \frac{P_{n}^{m}(x) P_{n+2 l}^{m}(x) d x}{1-x^{2}} \\
& \quad=(n+2 l-m+2) \int_{-1}^{1} \frac{P_{n}^{m}(x) P_{n+2 l+1}^{m-1}(x) d x}{\left(1-x^{2}\right)^{1 / 2}}, \text { where } l=0,1,2, \cdots .
\end{aligned}
$$

None of the integrals appearing in (2) can be calculated easily using the tables of integrals. Therefore, we shall seek to replace the associated Legendre function $P_{n}{ }^{m}(x)$ by its equivalent Rodrigues' formula defined by

$$
P_{k}^{m}(x)=\frac{\left(1-x^{2}\right)^{m / 2}}{2^{k} \cdot k !} \cdot \frac{d^{m+k}}{d x^{m+k}}\left(x^{2}-1\right)^{k}, \quad k=0,1,2, \cdots,
$$

and then perform repeated integration by parts. For this purpose, let us first consider the integral on the right-hand side of Eq. (2). This can be expressed in the following manner:

Received October 16, 1972.

AMS (MOS) subject classifications (1970). Primary 33A45.

Key words and phrases. Legendre function, integration by parts, recurrence relations, Rodrigues' formula, Leibnitz theorem for derivatives of products, Kronecker delta, orthogonality.

Copyright (c) 1974, American Mathematical Society 


$$
\int_{-1}^{1} \frac{P_{n}^{m}(x) P_{n+2 l+1}^{m-1}(x) d x}{\left(1-x^{2}\right)^{1 / 2}}=(-1)^{m-1} A_{l} /\left[2^{2 n+2 l+1} \cdot n !(n+2 l+1) !\right]
$$

where

$$
A_{l}=\int_{-1}^{1} d x\left[\frac{d^{n+2 l+m}}{d x^{n+2 l+m}}\left(x^{2}-1\right)^{n+2 l+1}\right] \cdot\left[\left(x^{2}-1\right)^{m-1} \cdot \frac{d^{n+m}}{d x^{n+m}}\left(x^{2}-1\right)^{n}\right]
$$

Since, at $x= \pm 1$, the quantities

$$
\frac{d^{n+m+2 l-i}}{d x^{n+m+2 l-i}}\left(x^{2}-1\right)^{n+2 l+1} \text { and } \frac{d^{i-1}}{d x^{i-1}}\left\{\left(x^{2}-1\right)^{m-1} \frac{d^{n+m}}{d x^{n+m}}\left(x^{2}-1\right)^{n}\right\}
$$

vanish for $m \leqq i$ and $m \geqq i+1$ respectively (with $i=0,1,2, \cdots),(n+m+2 l$ )-times repeated integrations by parts, starting with the integration of the first factor in the integrand of (4ii), result in the following expression for $A_{l}$ :

$$
A_{l}=(-1)^{n+m+2 l}
$$

$$
\cdot \int_{-1}^{1} d x\left(x^{2}-1\right)^{n+2 l+1} \cdot \frac{d^{n+m+2 l}}{d x^{n+m+2 l}}\left[\left(x^{2}-1\right)^{m-1} \cdot \frac{d^{n+m}}{d x^{n+m}}\left(x^{2}-1\right)^{n}\right] .
$$

By employing the Leibnitz theorem for derivatives of products, it can be shown that $A_{l}=0$, for all $l=0,1,2, \cdots$. Thus it is proved that

$$
\int_{-1}^{1} \frac{P_{n}^{m}(x) P_{n+2 l+1}^{m-1}(x) d x}{\left(1-x^{2}\right)^{1 / 2}}=0, \quad l=0,1,2, \cdots .
$$

Then, from (2) and (6), we have the following result:

$$
I_{l}=\hat{I}_{l}, \quad l=0,1,2, \cdots,
$$

where

$$
\begin{aligned}
& I_{l}=\int_{-1}^{1} \frac{x P_{n}^{m}(x) P_{n+2 l+1}^{m}(x) d x}{1-x^{2}}, \\
& \hat{I}_{l}=\int_{-1}^{1} \frac{P_{n}^{m}(x) P_{n+2 l}^{m}(x) d x}{1-x^{2}} .
\end{aligned}
$$

With the aid of Eqs. (1) and (6), the following relations can be established:

$$
\hat{I}_{l+1}=\hat{I}_{l}, \quad l=0,1,2, \cdots,
$$

i.e.

$$
\hat{I}_{0}=\hat{I}_{1}=\hat{I}_{2}=\cdots=\hat{I}_{l}=\cdots .
$$

Similarly, one finds also (using (7i) and (8i))

$$
I_{0}=I_{1}=I_{2}=\cdots=I_{l}=\cdots .
$$

In view of the following recurrence relation

$$
\left(1-x^{2}\right)^{1 / 2} \cdot P_{k}^{m+1}(x)=(k+m) P_{k-1}^{m}(x)-(k-m) x P_{k}^{m}(x),
$$

and the equality (7i), we have

$$
I_{l}=\hat{I}_{l}=\hat{\hat{I}}_{l} / 2 m, \quad m \neq 0
$$


where

$$
\hat{\hat{I}}_{l}=\int_{-1}^{1} \frac{P_{n}^{m}(x) P_{n+2 l+1}^{m+1}(x) d x}{\left(1-x^{2}\right)^{1 / 2}}, \quad l=0,1,2, \cdots .
$$

Let us now define the following quantities:

$$
\begin{aligned}
& J_{l}=\int_{-1}^{1} x P_{n}^{m}(x) P_{n-2 l-1}^{m}(x) /\left(1-x^{2}\right) \cdot d x, \\
& \hat{J}_{l}=\int_{-1}^{1} d x P_{n}^{m}(x) P_{n-2 l}^{m}(x) /\left(1-x^{2}\right), \\
& \hat{\hat{J}}^{l}=\int_{-1}^{1} d x P_{n}^{m}(x) P_{n-2 l-1}^{m-1}(x) /\left(1-x^{2}\right)^{1 / 2}
\end{aligned}
$$

Now employing the relations

$$
\left(1-x^{2}\right)^{1 / 2} \cdot P_{k}^{m+1}(x)=(k+m+1) x P_{k}^{m}(x)-(k-m+1) P_{k+1}^{m}(x),
$$

and

$$
\left(1-x^{2}\right)^{1 / 2} \cdot P_{k}^{m-1}(x)=\left[P_{k+1}^{m}(x)-x P_{k}^{m}(x)\right] /(k+m),
$$

one can easily obtain the following results:

$$
\frac{(n+m-2 l)}{(n-m-2 l)} J_{l}-\hat{J}_{l}=\frac{1}{(n-m-2 l)} \int_{-1}^{1} \frac{P_{n}^{m}(x) P_{n-2 l-1}^{m+1}(x) d x}{\left(1-x^{2}\right)^{1 / 2}},
$$

and

(14ii) $\quad \hat{J}_{l}-J_{l}=(n+m-2 l-1) \hat{\hat{J}}_{l}, \quad(n-m-1) / 2 \geqq l \geqq 0$.

By appropriate choice of indices, $n, m$ and $l$, it follows from (6), that

$$
\int_{-1}^{1} \frac{P_{n}^{m}(x) P_{n-2 l-1}^{m+1}(x) d x}{\left(1-x^{2}\right)^{1 / 2}}=0
$$

Then the relations (14i) and (14ii) reduce to

$$
J_{l}=\frac{(n-m-2 l)}{(n+m-2 l)} \hat{J}_{l}=\frac{(n+m-2 l-1)(n-m-2 l)}{2 m} \hat{\hat{J}}_{l}, \quad m \neq 0 .
$$

3. A Theorem. We now prove that, for integer values of $n, m$ and $s$,

$$
F_{s}=\int_{-1}^{1} \frac{x}{1-x^{2}} \cdot P_{n}^{m}(x) P_{s}^{m}(x) d x
$$

$$
=\left\{\begin{array}{rr}
((n+m) ! /[m(n-m) !]) \delta_{s, n+2 l+1}, & \text { if } s>n, \\
((n+m-2 l-1) ! /[m(n-m-2 l-1) !]) \delta_{s, n-2 l-1}, & \text { if } s<n, \\
l=0,1,2, \cdots, & \text { and } m \neq 0,
\end{array}\right.
$$

where the Kronecker delta function $\delta_{i j}$ has its usual meaning.

Let us consider that $m$ and $n(>m)$ are fixed and $s$ is a variable index. If $s=$ $n \pm 2 l$, then the integrand becomes an odd function of $x$ and consequently the integral 
vanishes. However, the integral does not vanish for $s=n \pm(2 l+1)$. This establishes the validity of the introduction of the Kronecker delta function in (17i) and (17ii). In order to compute the integral for various $s$, let us first take $s=n+1$ (i.e., $l=0$ ) and replace the associated Legendre functions by the Rodrigues' formula (3). Then we have

$$
\begin{aligned}
F_{n+1}=\int_{-1}^{1} x P_{n}^{m}(x) P_{n+1}^{m}(x) /\left(1-x^{2}\right) d x=\frac{(-1)^{m-1}}{2^{2 n+1} \cdot n !(n+1) !} \\
\cdot \int_{-1}^{1} x\left(x^{2}-1\right)^{m-1} \cdot \frac{d^{n+m+1}}{d x^{n+m+1}}\left(x^{2}-1\right)^{n+1} \cdot \frac{d^{n+m}}{d x^{n+m}}\left(x^{2}-1\right)^{n} d x .
\end{aligned}
$$

Writing $x\left(x^{2}-1\right)^{m-1}=(1 / 2 m)\left(d\left(x^{2}-1\right)^{m} / d x\right), m \neq 0$, and then integrating by parts once, we have, from (18i),

$$
F_{n+1}=\frac{(-1)^{m}}{2^{2 n+2} \cdot m \cdot n !(n+1) !} \cdot\left[g_{1}+g_{2}\right],
$$

where

$$
g_{1}=\int_{-1}^{1}\left(x^{2}-1\right)^{m} \cdot \frac{d^{n+m+2}}{d x^{n+m+2}}\left(x^{2}-1\right)^{n+1} \cdot \frac{d^{n+m}}{d x^{n+m}}\left(x^{2}-1\right)^{n} d x
$$

and

(18iv) $\quad g_{2}=\int_{-1}^{1}\left(x^{2}-1\right)^{m} \cdot \frac{d^{n+m+1}}{d x^{n+m+1}}\left(x^{2}-1\right)^{n+1} \cdot \frac{d^{n+m+1}}{d x^{n+m+1}}\left(x^{2}-1\right)^{n} d x$.

For the integral $g_{1}$, integrating by parts $(n+m)$-times starting with the integration of the factor $d^{n+m}\left(x^{2}-1\right)^{n} / d x^{n+m}$ and then using in the resulting expression the Leibnitz theorem for derivatives of products, we have

$$
g_{1}=(-1)^{n+m} \cdot \sum_{j=0}^{n+m} \frac{(n+m) !}{j !(n+m-j) !}
$$

$$
\cdot \int_{-1}^{1} d x\left(x^{2}-1\right)^{n}\left\{\frac{d^{n+m-j}}{d x^{n+m-j}}\left(x^{2}-1\right)^{m}\right\}\left\{\frac{d^{n+m+2+j}}{d x^{n+m+2+j}}\left(x^{2}-1\right)^{n+1}\right\} .
$$

A little effort will show that all the terms of the above series in (18v) vanish except for $j=n-m$. Thus the contribution for $j=n-m$ can be shown to be

$$
g_{1}=(-1)^{m} \cdot 2^{2 n+2} \cdot n !(n+1) !(n+m) ! /(n-m) ! .
$$

For the integral in (18iv), we apply again repeated integrations by parts $(n+m+$ 1)-times starting with the integration of the factor $d^{n+m+1}\left(x^{2}-1\right)^{n+1} / d x^{n+m+1}$. This procedure shows that $\mathfrak{g}_{2}=0$. If now the Rodrigues' formula (3) is used in (18iv) to replace the derivatives of $\left(x^{2}-1\right)^{n+1}$ and $\left(x^{2}-1\right)^{n}$ by appropriate Legendre functions, then vanishing of $g_{2}$ can be shown to be equivalent to the following relation:

$$
\int_{-1}^{1} \frac{P_{n+1}^{m}(x) P_{n}^{m+1}(x)}{\left(1-x^{2}\right)^{1 / 2}} d x=0=g_{2}
$$

It turns out that the result in (20) is a special case of the relation (15). In view of (19) and (20), we have from (18i) and (18ii) the following result: 


$$
F_{n+1}=\int_{-1}^{1} \frac{x}{1-x^{2}} \cdot P_{n}^{m}(x) P_{n+1}^{m}(x) d x=\frac{(n+m) !}{m(n-m) !} .
$$

Comparing now (21) and (7ii), one finds $F_{n+1}=I_{0}$. Furthermore, $F_{0}=I_{l}$ for $s>n$. When these relations are combined with the result in (9), we have

$$
F_{s}=I_{l}=I_{0}=F_{n+1}=(n+m) ! / m(n-m) !, \quad s>n,
$$

which is the result desired in the first part [Eq. (17i)] of the theorem. The second half [Eq. (17ii)] of the theorem can be proved in a similar manner or it can be derived from (17i) by a judicious change of various indices. Thus one finds

$$
F_{s}=J_{l}=\frac{(n+m-2 l-1) !}{m(n-m-2 l-1) !}, \quad s<n .
$$

Similarly, in view of the relations (7i), (8i), (16), (17i) and (17ii), one may also write

$$
\begin{aligned}
\int_{-1}^{1} \frac{P_{n}^{m}(x) P_{s}^{m}(x) d x}{1-x^{2}}=\left\{\begin{array}{lr}
\frac{(n+m) !}{m(n-m) !} \delta_{s, n+2 l}, & \text { if } s \geqq n,
\end{array}\right. \\
=\left\{\begin{array}{rr}
\frac{(n+m-2 l) !}{m(n-m-2 l) !} \delta_{s, n-2 l}, & \text { if } s \leqq n, \\
& l=0,1,2, \cdots .
\end{array}\right.
\end{aligned}
$$

In [2], the above result is stated as

$$
\int_{-1}^{1} \frac{P_{n}^{m}(x) P_{n}^{k}(x)}{1-x^{2}} d x=\frac{(n+m) !}{m(n-m) !} \delta_{k, m},
$$

which is true as it stands. However, Jones [4] in his book presents it in the following manner

$$
\int_{-1}^{1} \frac{P_{n}^{m}(x) P_{s}^{m}(x) d x}{1-x^{2}}=\frac{(n+m) !}{m(n-m) !} \delta_{s, n},
$$

which is not correct in view of the results given here in (24i) and (24ii).

Another integral which was also used in [1] appears in Hobson's book [3] incorrectly as an exercise. This is

$$
\int_{-1}^{1} x P_{n}^{m}(x) P_{l}^{m}(x) d x
$$

This is not a difficult integral to evaluate. Using the last two expressions in Eq. (1) to replace $x P_{n}{ }^{m}(x)$ and the well-known ([2], [3]) result

$$
\int_{-1}^{1} P_{n}^{m}(x) P_{s}^{m}(x) d x=\frac{2}{2 n+1} \cdot \frac{(n+m) !}{(n-m) !} \delta_{s, n},
$$

it can easily be shown that

$$
\begin{aligned}
\int_{-1}^{1} x P_{n}^{m}(x) & P_{l}^{m}(x) d x \\
& =\frac{2(l+m+1) ! \delta_{n, l+1}}{(2 l+1)(2 l+3)(l-m) !}+\frac{2(l+m) ! \delta_{n, l-1}}{(2 l-1)(2 l+1)(l-m-1) !} .
\end{aligned}
$$


4. Additional Results. In this section, we present a number of results, without offering any proof since these results can be obtained from those given in Sections 2 and 3 together with some well-known relations. For $l=0,1,2, \cdots$, we have

$$
\begin{aligned}
& \int_{-1}^{1} \frac{P_{n}^{m}(x) P_{n+2 l+1}^{m+1}(x) d x}{\left(1-x^{2}\right)^{1 / 2}}=\frac{2(n+m) !}{(n-m) !}, \\
& \int_{-1}^{1} \frac{P_{n}^{m+1}(x) P_{n+2 l+1}^{m}(x) d x}{\left(1-x^{2}\right)^{1 / 2}}=0 \text {, } \\
& \int_{-1}^{1} \frac{P_{n-1}^{m}(x) P_{n-2 l}^{m+1}(x) d x}{\left(1-x^{2}\right)^{1 / 2}}=0, \\
& \int_{-1}^{1} \frac{P_{n}^{m}(x) P_{n-2 l-1}^{m-1}(x) d x}{\left(1-x^{2}\right)^{1 / 2}}=\frac{2(n+m-2 l-2) !}{(n-m-2 l) !}, \\
& \int_{-1}^{1} P_{n+2 l+1}^{m}(x) \frac{d}{d x} P_{n}^{m}(x) d x=\delta_{0, m}-\frac{(n+m) !}{(n-m)}, \\
& \int_{-1}^{1} P_{n}^{m}(x) \frac{d}{d x} P_{n+2 l+1}^{m}(x) d x=\delta_{0, m}+\frac{(n+m) !}{(n-m) !}, \\
& \int_{-1}^{1} P_{n}^{m}(x) \frac{d}{d x} P_{n-2 l-1}^{m}(x) d x=\delta_{0, m}-\frac{(n-2 l-1+m) !}{(n-2 l-1-m) !}, \\
& \int_{-1}^{1} P_{n-2 l-1}^{m}(x) \frac{d}{d x} P_{n}^{m}(x) d x=\delta_{0, m}+\frac{(n-2 l-1+m) !}{(n-2 l-1-m) !}, \\
& \int_{-1}^{1} x P_{n}^{m}(x) \frac{d}{d x} P_{n}^{m}(x) d x=\delta_{0, m}-\frac{(n+m) !}{(2 n+1)(n-m) !}, \\
& \int_{-1}^{1} x P_{n}^{m}(x) \frac{d}{d x} P_{n \pm 2 l \pm 1}^{m}(x) d x=0, \\
& \int_{-1}^{1} x P_{n \pm 2 l \pm 1}^{m}(x) \frac{d}{d x} P_{n}^{m}(x) d x=0, \\
& \int_{-1}^{1}\left(1-x^{2}\right) P_{l}^{m}(x) \frac{d}{d x} \cdot P_{n}^{m}(x) d x=\left[\frac{2(l+1)(l+m+1) !}{(2 l+1)(2 l+3)(l-m) !} \delta_{n, l+1}\right. \\
& \left.-\frac{2(l-1)(l-m) !}{(2 l-1)(2 l+1)(l-m-1) !} \delta_{n, l-1}\right] \text {, } \\
& \int_{-1}^{1}\left(1-x^{2}\right)^{2} \frac{d}{d x} P_{n}^{m}(x) \frac{d}{d x} P_{l}^{m}(x) d x=\frac{2(l+m) !}{(2 l+1)^{2}(l-m) !} \\
& \cdot\left\{\frac{(l+1)^{2}(l+m)(l-m)}{2 l-1}+\frac{l^{2}(l-m+1)(l+m+1)}{2 l+3}\right\} \delta_{n, l} \\
& -\frac{2 l(l+3)(l+m+2) ! \delta_{n, l+2}}{(2 l+1)(2 l+3)(2 l+5)(l-m) !} \\
& -\frac{2(l-2)(l+1)(l+m) ! \delta_{n, l-2}}{(2 l-3)(2 l-1)(2 l+1)(l-m-2) !} \text {. }
\end{aligned}
$$

For $l=1,2,3, \cdots$, we have the following relations: 


$$
\begin{aligned}
& \int_{-1}^{1} x P_{n}^{m}(x) \frac{d}{d x} P_{n+2 l}^{m}(x) d x=\delta_{0, m}+\frac{(n+m) !}{(n-m) !} \\
& \int_{-1}^{1} x P_{n}^{m}(x) \frac{d}{d x} P_{n-2 l}^{m}(x) d x=\delta_{0, m}-\frac{(n-2 l+m) !}{(n-m-2 l) !}, \\
& \int_{-1}^{1} x P_{n+2 l}^{m}(x) \frac{d}{d x} P_{n}^{m}(x) d x=\delta_{0, m}-\frac{(n+m) !}{(n-m) !} \\
& \int_{-1}^{1} x P_{n-2 l}^{m}(x) \frac{d}{d x} P_{n}^{m}(x) d x=\delta_{0, m}+\frac{(n-2 l+m) !}{(n-m-2 l) !}
\end{aligned}
$$

It may be noted that one of the referees brought to the author's knowledge some related work by Power [5]. For this, the author is thankful to the referee. It appears that any related results Power presented turn out to be respective special cases of the author's. For instance, Power's equations $(\mathrm{j}),(\mathrm{k})$ and $(\mathrm{m})$ are the special cases of the results presented here in (29), (6) and (24), respectively.

Cornell Aeronautical Laboratory, Inc.*

P. O. Box 235

Buffalo, New York 14221

1. S. N. Samaddar, Scattering of Cylindrical Waves by a Spherical Object, Proc. 1971 International Sympos. Antennas and Propagation, Sendai, Japan, Sept. 1-3, 1971, p. 195.

2. P. M. Morse \& H. Feshback, Methods of Theoretical Physics. Vol. II, Chap. 11, McGraw-Hill, New York, 1953. MR 15, 583.

3. E. W. HoBson, The Theory of Spherical and Ellipsoidal Harmonies, Chelsea, New York, 1955. MR 16, 356.

4. D. S. JONES, The Theory of Electromagnetism, Internat. Ser. of Monographs on Pure and Appl. Math., vol. 47, Macmillan, New York, 1964. MR 28 \#4759.

5. G. Power, "The associated Legendre polynomial," Math. Gaz., v. 38, 1954, pp. 115116. MR 15, 701 .

* Present name: CalsPaN Corporation. 